\title{
INFORMES*
}

\section{El Decreto 22/2007, de 30 de enero, por el que se establece el marco regulador de las ayudas a favor del medio ambiente que se concedan por la Administración de la Junta de Andalucía**}

I.- El Decreto 22/2007 regula las ayudas que puedan conceder la Administración de la Junta de Andalucía, sus organismos autónomos y empresas en el ámbito de sus competencias, que tengan como finalidad la protección del medio ambiente en Andalucía y que se dirijan a proyectos que se realicen en todos los campos de la actividad económica, excepto el sector agrario (art. 1.1).

El Decreto deroga al anterior sobre la materia, el 23/2001, de 13 de febrero, del que es reproducción fiel, salvo en lo que concierne a la definición de pequeña y mediana empresa ${ }^{1}$; a la adición de un segundo párrafo en el art. 3 y de otras especificaciones relativas a los sujetos que se enumeran en dicho art. 3.2; a la previsión expresa de ayudas de minimis; a la mención de la Ley 38/2003, de 17 de noviembre, General de Subvenciones, que el Decreto anterior, lógicamente, no pudo tener en cuenta; y a la exigencia de informe favorable de la Secretaría General de Acción Exterior de la Consejería de la Presidencia antes de la aprobación de las normas de adaptación que pudieran ser necesarias.

Para situar el Decreto en su debido contexto, conviene tener presente que en el ámbito de las ayudas públicas, como es sabido, incide lo dispuesto en el art. 87 del Tratado Constitutivo de la Comunidad Europea, que sienta como regla general en la materia la de la incompatibilidad con el mercado

\footnotetext{
* Sección redactada bajo la dirección de José Ignacio Morillo-Velarde Pérez.

** Realizado por María del Carmen Núñez Lozano, profesora titular de Derecho Administrativo de la Universidad de Huelva.

${ }^{1} \mathrm{Al}$ haberse sustituido la Recomendación 96/280/CE por la Recomendación 2003/361/CE.
} 
común, en la medida en que afecten a los intercambios comerciales entre Estados miembros, de aquellas ayudas que falseen o amenacen falsear la competencia, favoreciendo a determinadas empresas o producciones (ap. 1). El precepto, no obstante, relaciona una serie de ayudas que son compatibles (ap. 2) y señala otras que pueden considerarse compatibles (ap. 3), de entre las cuáles menciona expresamente la Exposición de Motivos del Decreto 22/2007 las que tienen como objetivo específico fomentar el desarrollo económico de regiones en las que el nivel de vida sea anormalmente bajo, la realización de un proyecto importante de interés común europeo, así como el desarrollo de determinadas actividades, siempre que no alteren las condiciones de los intercambios en forma contraria al interés común. Estas ayudas, pues, han de ser supervisadas por la Comisión, para lo cuál debe ser informada de los proyectos dirigidos a conceder o modificar ayudas con la suficiente antelación para poder presentar sus observaciones (art. 88.3 TCE); sus criterios al efecto, respecto de las que persiguen la protección del medio ambiente, son los que se contienen en las Directrices comunitarias sobre ayudas estatales a favor del medio ambiente (2001/C 37/03, Diario Oficial de las Comunidades Europeas de 3 de febrero) ${ }^{2}$.

Siguiendo las pautas de las referidas Directrices se ha aprobado el Decreto que comentamos ${ }^{3}$ que, como se ha anticipado, viene a reproducir el anterior de 2001, con las salvedades expresadas, singularmente las que conciernen a las ayudas a los sujetos enumerados en el art. 3.2, que no se consideran ayudas de estado, y a las ayudas de minimis. Por ello, cabe concluir que la finalidad perseguida con el Decreto es, de suyo, renovar la autorización

\footnotetext{
${ }^{2}$ Estas Directrices sustituyeron a las aprobadas en 1994, que expiraron el 31 de diciembre de 1999 y que fueron prorrogadas, no obstante, hasta el 31 de diciembre de 2000.

Está prevista su aplicación hasta 31 de diciembre de 2007. En la síntesis que ofrece la Exposición de Motivos del Decreto que comentamos, "su finalidad es garantizar la protección del medio ambiente y el desarrollo sostenible sin producir efectos desproporcionados en la competencia, respetando los principios de «quien contamina paga» y de integración de los costes relativos a la protección medioambiental en los costes de producción, y estimulando a las empresas para que realicen un esfuerzo de protección medioambiental superior al que les exige la normativa de aplicación. Asimismo, se incluyen las intervenciones a favor de un uso racional de la energía y de la utilización de las energías renovables, debido a las importantes ventajas que ofrecen para el medio ambiente".

${ }^{3}$ Lo que explica el que excluya de su ámbito de aplicación el sector agrario, que también se excluye de estas Directrices, por estar contemplado en las Directrices comunitarias sobre ayudas estatales al sector agrario (DO C 28 de 1 de febrero de 2000).
} 
de la Comisión Europea para las ayudas que ya estaban contempladas en el Decreto 23/2001 y la de establecer un texto único sobre el marco regulador de las ayudas a favor del medio ambiente que a la par contemple ciertas singularidades que atañen a esas dos clases de ayudas antes mencionadas ${ }^{4}$.

II.- Pueden ser beneficiarios de las ayudas las personas físicas o jurídicas, públicas o privadas, o sus agrupaciones, radicadas en Andalucía (art. 3.1) ${ }^{5}$. De entre ellas, el Decreto menciona específicamente, según se ha anticipado, a los Ayuntamientos, Diputaciones u otras Entidades Locales, Organismos Autónomos de naturaleza administrativa, Entidades Públicas, Agencias, Instituciones, Consorcios y Asociaciones de carácter público cuando no ejerzan la actividad económica objeto de ayuda, las Entidades gestoras y Servicios de la Seguridad Social, las instituciones sin ánimo de lucro y las personas físicas que no ejerzan actividad económica, con la finalidad de precisar que las ayudas que se les conceda "están excluidas del régimen de ayudas de Estado por la propia naturaleza del beneficiario, por lo que no tienen carácter de ayuda de estado" (art. 3.2).

III.- En cuanto a las actividades que se pueden subvencionar, el Decreto especifica los objetivos que han de perseguir las ayudas (art. 2) y, en relación con cada uno de ellos, los objetos que correlativamente han de tener (art. 4), así como los correspondientes proyectos que se pueden subvencionar (art. 5$)^{6}$.

\footnotetext{
${ }^{4} \mathrm{El}$ art. 1.4 señala que las disposiciones del Decreto "se entenderán sin perjuicio de lo establecido en el Reglamento de la Comisión Europea relativo a las ayudas de mínimis". La omisión de los datos del Reglamento puede deberse a la trascripción del art. 1.4 del Decreto anterior, con sustitución de la referencia a "la Comunicación de la Comisión Europea relativa a las ayudas de mínimis" por esta otra del Reglamento. En el art. 7.3 del Decreto sí se cita correctamente el Reglamento.

${ }^{5}$ En el caso de que el beneficiario sea una pequeña y mediana empresa, se entiende como tal la que se define en la Recomendación 2003/361/CE de la Comisión, de 6 de mayo de 2003, sobre definición de microempresas, pequeñas y medianas empresas (art. 3.1). A tenor de la misma, la categoría de microempresas, pequeñas y medianas empresas (PYME) está constituida por las empresas que ocupan a menos de 250 personas y cuyo volumen de negocios anual no excede de 50 millones de euros o cuyo balance general anual no excede de 43 millones de euros; en la categoría de las PYME, se define a una pequeña empresa como una empresa que ocupa a menos de 50 personas y cuyo volumen de negocios anual o cuyo balance general anual no supera los 10 millones de euros; en la categoría de las PYME, se define a una microempresa como una empresa que ocupa a menos de 10 personas y cuyo volumen de negocios anual o cuyo balance general anual no supera los 2 millones de euros.

${ }^{6} \mathrm{Si}$ bien se advierte que las normas específicas reguladoras de cada línea de ayudas podrán establecer restricciones sobre determinados proyectos conforme a las directrices de política económica (Disposición Final Segunda 2).
} 
Así, las ayudas deben perseguir al menos uno de los objetivos siguientes: a) El fomento de la inversión en infraestructuras e instalaciones destinadas a la protección del medio ambiente; b) La mejora del control ambiental de las pequeñas y medianas empresas; c) El ahorro energético; d) La promoción del uso de las energías renovables; e) La mejora del espacio natural afectado por la industria extractiva (art. 2).

Las ayudas que persigan el fomento de la inversión en infraestructuras e instalaciones destinadas a la protección del medio ambiente deben tener como objeto las inversiones destinadas a superar el nivel de protección exigido por la normativa comunitaria (art. 4.1). Se consideran proyectos subvencionables: a) Los proyectos de inversión destinada a superar el nivel de protección exigido por la normativa comunitaria: inversiones realizadas en cumplimiento de normas nacionales más estrictas que las comunitarias y que permiten alcanzar un mayor nivel de protección ambiental e inversiones realizadas para la protección del medio ambiente superando las normas comunitarias o en ausencia de tales normas. b) Los estudios de viabilidad técnico/económica y asesoramiento externo en la fase de planificación y de ejecución material de la inversión, exclusivamente para las pequeñas y medianas empresas. c) Los proyectos destinados a subsanar daños ambientales, siempre que no sea posible identificar al responsable o atribuirle jurídicamente la responsabilidad económica (art. 5.1).

El objeto de las ayudas que tengan como objetivo la mejora del control ambiental de las pequeñas y medianas empresas ha de ser la puesta en marcha de iniciativas relacionadas con el control de los efectos ambientales de las actividades productivas (art. 4.2). Se consideran proyectos subvencionables: a) La acreditación, por una sola vez, del cumplimiento por las Entidades Colaboradoras de la Consejería de Medio Ambiente en materia de protección ambiental, de los «Criterios generales para el funcionamiento de los diversos tipos de organismos que realizan inspección», contemplados en la normativa UNE-EN correspondiente. b) La acreditación, por una sola vez, del cumplimiento por los laboratorios de ensayo que desarrollen su actividad en el sector medioambiental, de los «Criterios generales para el funcionamiento de los laboratorios de ensayo", contemplados en la normativa UNE-EN correspondiente. c) El cumplimiento de las normas anteriores por las empresas que estén obligadas a realizar autocontroles. d) La certificación, por una sola vez, por Entidades Colaboradoras de la Consejería de Medio Ambiente de los equipos de medición automática en continuo instalados en las empresas. e) La implantación de sistemas de gestión y auditoría medioambiental en las empresas. f) La acreditación, por una sola vez, de empresas verificadoras 
medioambientales, de conformidad con las exigencias de la normativa comunitaria que permita a las empresas adherirse con carácter voluntario a un sistema comunitario de gestión y auditoría ambientales. g) La divulgación e información, realización de diagnósticos y estudios sectoriales encaminados a la mejora del control ambiental. h) La obtención, por una sola vez, de la etiqueta ecológica de acuerdo con la normativa aplicable (art. 5.2).

Según el art. 4.3, las ayudas que tengan como objetivo el ahorro energético, deben tener como objeto las inversiones para adecuar las instalaciones, productos y procesos productivos a fin de conseguir beneficios para el medio ambiente y/o mejorar su eficiencia energética, así como las instalaciones de cogeneración con producción térmica para diferentes usos y producción eléctrica. Para los beneficiarios que cita el art. 3.2, las ayudas al ahorro energético también tendrán por objeto la adquisición de vehículos limpios o que utilicen combustibles alternativos, gas natural, hidrógeno, tecnologías que incluyan sistemas eléctricos, o sistemas híbridos (combustión/eléctricos). Son proyectos subvencionables, según el art. 5.3, los siguientes: a) Auditorías energéticas en instalaciones existentes e implantación de las recomendaciones, exclusivamente en las pequeñas y medianas empresas. En el caso de empresas que no tengan esta consideración, sólo se pueden conceder estas ayudas con carácter de minimis, respetando las condiciones señaladas en el artículo 7.3. b) Estudios de viabilidad de proyectos de ahorro energético como parte integral de un proyecto y su implantación, exclusivamente en las pequeñas y medianas empresas. En el caso de empresas que no tengan esta consideración sólo se pueden conceder estas ayudas con carácter de minimis, respetando las condiciones señaladas en el artículo 7.3. c) Proyectos de instalaciones que generen simultáneamente energía térmica y eléctrica (cogeneración), que cumplan los requisitos establecidos por la normativa vigente para poder acogerse al régimen especial de producción eléctrica y que supongan un ahorro de al menos el 10\% de energía y reduzcan la situación de contaminación ambiental. d) Proyectos de adecuación o sustitución de instalaciones, equipos o procesos existentes, de modo que supongan un ahorro de al menos el $10 \%$ de energía y reduzcan la situación de contaminación ambiental, en relación con la situación anterior. e) Proyectos de adecuación de instalaciones y equipos que permitan la sustitución de combustibles o energías tradicionales por gas natural, siempre que esta sustitución vaya acompañada de un ahorro energético de al menos el $5 \%$ y se reduzca la situación de contaminación ambiental. f) La adquisición de vehículos limpios o que utilicen combustibles alternativos, con gas natural o hidrógeno o con tecnologías que incluyan sistemas eléctricos o híbridos (combustión/eléctricos), exclusivamente para los beneficiarios del art. 3.2. 
Las ayudas que persigan la promoción del uso de las energías renovables han de tener como objeto las inversiones en plantas de producción de energía con recursos energéticos renovables y en elementos de transformación de fuentes energéticas renovables (art. 4.4). Los proyectos subvencionables son: a) Estudios de viabilidad de proyectos de diversificación energética como parte integral de un proyecto y su implantación, siempre que comporten un ahorro energético del $10 \%$ y reduzcan la situación de contaminación ambiental, y exclusivamente para las pequeñas y medianas empresas. b) Inversiones en instalaciones centralizadas de aprovechamiento de energías renovables, con producción de energía eléctrica evacuada a la red. c) Inversiones que contemplen la diversificación energética, siempre que comporten un ahorro energético del $10 \%$ y reduzcan la situación de contaminación ambiental. d) Inversiones en elementos de transformación de fuentes energéticas renovables. e) Inversiones en instalaciones de aprovechamiento de energías renovables para utilización directa por los usuarios (art. 5.4).

Finalmente, el objeto de las ayudas que tengan como objetivo la mejora del espacio natural afectado por la industria extractiva son las inversiones en acciones de cuya ejecución se derive la mejora medioambiental del espacio natural afectado por la industria extractiva (art. 4.5). Se consideran subvencionables los siguientes proyectos: a) Inversiones para la disminución del deterioro que la actividad extractiva causa al medio ambiente. b) Inversiones para la restauración de los terrenos afectados por antiguas actividades mineras o en proceso de clausura y abandono autorizado por la Administración competente. c) Aprovechamiento de residuos mineros ubicados en escombreras, depósitos de residuos, presas, balsas y diques que permitan restituir el medio natural afectado por los mismos (art. 5.5).

A su vez, los proyectos subvencionables han de reunir los siguientes requisitos: a) Viabilidad desde el punto de vista técnico, económico, financiero y medioambiental. b) El beneficiario debe disponer de las autorizaciones de carácter ambiental que le sean exigibles en función de su actividad. c) En los proyectos destinados a la realización de inversiones que tengan por finalidad la superación de las normas comunitarias por la existencia de normas nacionales más estrictas, las instalaciones deben estar en funcionamiento desde al menos dos años antes de la entrada en vigor de las nuevas normas nacionales (art. 7.1). Cuando se trate de la adquisición de patentes, licencias de explotación o de conocimientos técnicos patentados, deben reunirse además estos otros requisitos: a) Ser considerados elementos del activo amortizables. b) Ser 
adquiridos en condiciones de mercado a empresas con las que el adquirente no tenga vínculos financieros. c) Figurar en el activo de la empresa y permanecer en el establecimiento del beneficiario de la ayuda durante un período mínimo de cinco años. En caso de reventa en el transcurso de los cinco años, el producto de la venta deberá deducirse de los costes subvencionables (art. 7.2).

En cada uno de los proyectos relacionados serán subvencionables los conceptos que se especifiquen en las normas que desarrollen el Decreto que comentamos y en las convocatorias respectivas, a partir de la enumeración que contiene el art. 6.1, que es la siguiente: a) La adquisición de los terrenos necesarios para la implantación del proyecto. b) La urbanización y obras exteriores adecuadas a las necesidades del proyecto. c) Las traídas y acometidas de servicios. d) La edificación de obra civil vinculada al proyecto. e) La maquinaria, instalaciones, materias primas y los bienes de equipos necesarios. Para los proyectos del artículo 5.3.f), serán conceptos subvencionables las unidades propulsoras eficientes de vehículos. f) En el sector del transporte se exceptúa la adquisición de activos móviles ${ }^{7}$. g) Otras inversiones en activos fijos materiales. h) Servicios externos para la elaboración de proyectos, los estudios de viabilidad o de diagnóstico, y otros gastos de asesoramiento externo. i) Los gastos de certificación o acreditación. j) Los trabajos de planificación, ingeniería de proyecto y dirección facultativa de los trabajos. k) Inversiones en activos fijos inmateriales para la gestión medioambiental. 1) Los costes de las auditorías necesarias para justificar el cumplimiento de condiciones.

Lógicamente, los costes subvencionables se limitan estrictamente al coste adicional necesario para alcanzar los objetivos medioambientales, de modo que se excluyen los gastos por inversiones en terrenos u obra civil que no estén directamente vinculados a la protección del medio ambiente, los elementos de transporte y equipos informáticos destinados a funciones económico/administrativas dentro de la empresa, y los gastos generales de inversión que no estén en relación con la protección del medio ambiente (art. 6.2). Por otra parte, no es tampoco subvencionable entre los conceptos de inversión el Impuesto sobre el Valor Añadido recuperable (art. 6.4), ni los gastos financieros como consecuencia de la inversión, excepto en el caso de la bonificación de intereses en las ayudas a la inversión (art. 6.5), ni las inversiones e equipos usados, a no ser que se adquieran en condiciones de mercado y siempre deduciendo aquellos activos para cuya adquisición el transmitente

\footnotetext{
7 Esta letra f) parece ser una errata. La excepción debió contemplarse en la letra e), como hacía el Decreto 23/2001.
} 
hubiera recibido ayudas (art. 6.6). Ha de tenerse en cuenta además que para determinar los costes subvencionables de los proyectos dirigidos a la subsanación de daños ambientales debe tomarse en consideración el precio de adquisición del terreno por la empresa beneficiaria de la ayuda y el valor de dicho terreno después de su rehabilitación (art. 6.3). En cualquier caso, las normas específicas reguladoras de cada línea de ayudas podrán establecer restricciones sobre determinados conceptos subvencionables conforme a las directrices de política económica (Disposición Final Segunda 2).

IV.- La concesión de las ayudas requiere la aprobación y la publicación por los órganos competentes de normas específicas y convocatorias, aunque se pueden conceder subvenciones excepcionales o contraer compromisos jurídicos con los beneficiarios mediante resoluciones o convenios (Disposición Final Segunda 1). En la concesión se han de tener en cuenta las directrices sectoriales específicas y demás normas y criterios de la Unión Europea sobre sectores considerados sensibles (art. 1.3).

Las clases de ayudas que contempla el Decreto son: a) Subvenciones a la inversión. b) Subvenciones para financiar la prestación de servicios externos. c) Bonificación al tipo de interés de préstamos concertados con entidades financieras. d) Participación en forma de capital en el caso de las instalaciones de energía renovable o cogeneración. e) Préstamos y ayudas reembolsables para la realización de inversiones en activos fijos (art. 8.1).

Corresponde a las normas específicas que se dicten y a las correspondientes convocatorias la determinación de la intensidad de la ayuda, en términos brutos y mediante porcentajes enteros, dentro de los límites que prevé el propio Decreto (art. 8.2). En este sentido, la cuantía de las ayudas concedidas a un mismo proyecto de inversión no puede sobrepasar los porcentajes máximos del coste de la inversión subvencionable que se recogen en el Anexo del Decreto; tales porcentajes, sin embargo pueden incrementarse en el caso de ayudas a pequeñas y medianas empresas en los supuestos previstos en la normativa comunitaria y en el porcentaje que se asimismo se establece en el referido Anexo (art. 1.2) ${ }^{8}$. Ha de precisarse, no obstante, que

\footnotetext{
${ }^{8}$ Los porcentajes máximos son los siguientes: 1) El 40\% bruto del coste de inversión subvencionable, en todo el territorio de la Comunidad Autónoma de Andalucía; puede incrementarse el porcentaje cuando se trate de pequeñas y medianas empresas hasta un $10 \%$ en términos brutos. 2) Para las ayudas dirigidas a la promoción de las energías renovables, el anterior porcentaje puede beneficiarse de una prima de 10 puntos en los siguientes casos: a.- La energía fotovoltaica, la energía eólica en zonas distantes de la red eléctrica existente que padezcan un clima muy frío,
} 
en la medida en que las ayudas a determinados sujetos no tienen el carácter de ayuda de estado, el art. 3.2 del Decreto dispone que las ayudas a los beneficiarios que cita $^{9}$ no están sometidas a "las intensidades señaladas en el Anexo del Decreto".

Y debe recordarse también que, como recoge el art. 7.3, en los proyectos subvencionables citados en el art. 5.3.a) y 5.3.b) que tengan como beneficiario a empresas que no tengan el carácter de pequeñas y medianas, las ayudas tienen el carácter de minimis, de modo que se sujetan al Reglamento 1998/2006, de la Comisión, de 15 de diciembre de 2006 y no les es aplicable el Anexo. No obstante, tales ayudas deben cumplir las siguientes condiciones, que traen causa del Reglamento citado: a) No se podrán conceder en los supuestos contemplados en el artículo 1.1 del Reglamento $1998 / 2006^{10}$. b)

caliente o polvoriento, situadas en el mar, en una isla o en una región rural periférica, y la energía producida a partir de la biomasa; b.- Las instalaciones de energías renovables que permitan el suministro a toda una comunidad. Tales primas son acumulables si se reúnen las condiciones establecidas. 3) Para las ayudas destinadas a las actividades de asesoramiento a pequeñas y medianas empresas, el porcentaje de las mismas puede alcanzar el $50 \%$ bruto del coste subvencionable.

Tales porcentajes pueden ser modificados por la Comisión Europea, en cuyo caso la Consejería de la Presidencia publicará las correspondientes modificaciones en el Boletín Oficial de la Junta de Andalucía (Disposición Final Primera).

\footnotetext{
${ }^{9}$ Esto es, los Ayuntamientos, Diputaciones u otras Entidades Locales, Organismos Autónomos de naturaleza administrativa, Entidades Públicas, Agencias, Instituciones, Consorcios y Asociaciones de carácter público cuando no ejerzan la actividad económica objeto de ayuda, las Entidades gestoras y Servicios de la Seguridad Social, las instituciones sin ánimo de lucro y las personas físicas que no ejerzan actividad económica.
}

${ }^{10}$ Se refiere a: A) Ayuda concedida a las empresas que operan en los sectores de la pesca y la acuicultura según se contemplan en el Reglamento (CE) no 104/2000 del Consejo; B) Ayuda concedida a las empresas que operan en la producción primaria de los productos agrícolas que figuran en la lista del anexo I del Tratado; C) Ayuda concedida a las empresas que operan en la transformación y comercialización de los productos agrícolas que figuran en la lista del anexo I del Tratado, en los siguientes casos: a) cuando el importe de la ayuda se determine en función del precio o de la cantidad de dichos productos adquiridos a productores primarios o comercializados por las empresas interesadas, b) cuando la ayuda dependa de que se repercuta total o parcialmente sobre los productores primarios agricultores); D) Ayuda a actividades relacionadas con la exportación a terceros países o Estados miembros, es decir la ayuda vinculada directamente a las cantidades exportadas, a la creación y funcionamiento de una red de distribución o a otros gastos de explotación vinculados a la actividad de exportación; E) Ayuda subordinada a un uso de bienes nacionales con preferencia sobre los bienes importados; F) Ayuda concedida a empresas activas en el sector del carbón, según se define en el Reglamento (CE) no 1407/2002; G) Ayuda para la adquisición de vehículos de transporte de mercancías por carretera concedida a empresas que realicen por cuenta ajena operaciones de transporte de mercancías por carretera; H) Ayuda concedida a empresas en crisis. 
Estas ayudas solamente podrán ser concedidas en forma de subvenciones y otros tipos de ayuda respecto de los cuales sea posible calcular previamente con exactitud el equivalente bruto de subvención de la ayuda sin necesidad de proceder a una evaluación del riesgo conforme al artículo 2.4 del Reglamento $1998 / 2006^{11}$. c) La ayuda total de minimis concedida a una empresa determinada no será superior a 200.000 euros durante cualquier período de tres ejercicios fiscales; este límite se aplicará independientemente de la forma de la ayuda o del objetivo perseguido e indistintamente de si la ayuda concedida por la Junta de Andalucía está financiada total o parcialmente mediante recursos de origen comunitario; el período se determinará tomando como referencia los ejercicios fiscales; cuando un importe global de ayuda supere este límite máximo dicha ayuda no podrá concederse ni siquiera para una fracción que no supere el citado límite máximo, ya sea en el momento de su concesión o en un período posterior. d) El límite máximo del apartado c) se expresa como subvención en efectivo; todas las cifras empleadas serán brutas, es decir, antes de cualquier deducción en concepto de fiscalidad o de otra carga; cuando se conceda una ayuda de cualquier forma distinta a la subvención, el importe de la ayuda será el equivalente bruto de subvención de la

\footnotetext{
${ }^{11}$ Son las que el Reglamento denomina ayudas transparentes y que enumera en el citado art. 2.4: A) En las ayudas consistentes en préstamos, cuando el equivalente bruto de subvención se haya calculado sobre la base de los tipos de interés de mercado aplicables en el momento de la subvención. B) La ayuda consistente en aportaciones de capital no se considera ayuda transparente, a menos que la cantidad total de la aportación no supere el límite máximo de minimis. C) La ayuda consistente en medidas de capital de riesgo no se considera ayuda transparente, a menos que el régimen de capital de riesgo en cuestión aporte capital solamente hasta el límite máximo de minimis para cada empresa beneficiaria. D) La ayuda individual concedida en virtud de un régimen de garantía a empresas que no sean empresas en crisis se considera ayuda transparente cuando la parte garantizada que subyace al préstamo concedido con arreglo al régimen en cuestión no es superior a 1500000 EUR por empresa. La ayuda individual concedida con arreglo a un régimen de garantía a favor de las empresas que operan en el sector del transporte por carretera que no sean empresas en crisis se trata como ayuda transparente cuando la parte garantizada del préstamo subyacente concedido con arreglo a dicho régimen no excede de 750000 EUR por empresa. Si la parte garantizada del préstamo subyacente sólo corresponde a una proporción determinada de este límite, se considera que el equivalente bruto de subvención de dicha garantía corresponde a la misma proporción del límite aplicable contemplado en el art. 2.2 del Reglamento. La garantía no debe ser superior al $80 \%$ del préstamo subyacente. Los regímenes de garantía también se consideran transparentes si (i) antes de la aplicación del régimen, la metodología para calcular el equivalente bruto de subvención de las garantías se acepta tras la notificación de dicha metodología a la Comisión conforme a otro Reglamento adoptado por la Comisión en el ámbito de las ayudas estatales y (ii) la metodología aprobada contempla explícitamente el tipo de garantías y el tipo de transacciones subyacentes de que se trata en el contexto de la aplicación del Reglamento.
} 
misma; las ayudas que se reciban en varios plazos se actualizarán a su valor en el momento de la concesión; el tipo de interés que se deberá utilizar a efectos de actualización y de cálculo del equivalente bruto de subvención será el tipo de referencia aplicable en el momento de la concesión; e) Cuando se vaya a conceder una de estas ayudas de minimis a una empresa, se obtendrá de la empresa una declaración escrita sobre cualquier otra ayuda de minimis u otra ayuda estatal recibida durante los dos ejercicios fiscales anteriores y durante el ejercicio fiscal en curso; en consecuencia, sólo se podrá conceder la nueva ayuda de minimis hasta haber comprobado que ello no incrementa el importe total de la ayuda de minimis recibida durante el período de referencia de tres años por encima del límite máximo establecido en el apartado c).

El régimen de compatibilidad de las ayudas se establece en el art. 10. La regla general es la de que las ayudas pueden ser compatibles con otras para la misma finalidad, siempre que el conjunto de todas las concedidas para un mismo proyecto no sobrepase los límites establecidos en cada caso (art. 10.1). Cuando los gastos subvencionables puedan acogerse a ayudas de finalidades distintas, la parte común quedará sujeta al límite más favorable de los regímenes de que se trate (art. 10.2). Las disposiciones que regulen cada ayuda y las convocatorias correspondientes deben expresar la compatibilidad, los límites máximos para cada proyecto y los mecanismos que garanticen el conocimiento de todas las ayudas solicitadas para el proyecto (art. 10.3). En todo caso, antes de formular la propuesta de resolución, han de comprobarse las ayudas concedidas y las que se tengan solicitadas para el mismo proyecto, de lo que debe quedar constancia en el expediente; en los supuestos de ayudas cofinanciadas por los Fondos Estructurales deberán cumplirse las normas que sobre participación y gestión financiera de los fondos establezca la Unión Europea (art. 10.4). Por último, las ayudas de minimis previstas en el artículo 7.3 son compatibles con las restantes ayudas previstas en el Decreto o con aquellas otras que se concedan con cargo a cualquier otro régimen autorizado por la Comisión Europea o amparadas por un Reglamento de exención por categorías; sin perjuicio de lo anterior, la ayuda de minimis no se acumulará con ninguna otra ayuda pública correspondiente a los mismos gastos subvencionables si dicha acumulación da lugar a una intensidad de ayuda superior a la establecida para las circunstancias concretas de cada caso en un Reglamento de Exención por categorías o en una Decisión adoptada por la Comisión (art. $10.5)^{12}$.

12 Vid. el art. 2.5 del Reglamento (CE) 1998/2006, de la Comisión, de 15 de diciembre de 2006. 
V.- Siguiendo lo establecido en el artículo 110 de la Ley 5/1983, de 19 de julio, General de la Hacienda Pública de la Comunidad Autónoma de Andalucía ${ }^{13}$, todo cambio sobre el proyecto original o sobre las condiciones que se tuvieron en cuenta para la concesión de la subvención , así como la obtención de ayudas otorgadas por otras Administraciones o entes públicos o privados, nacionales o internacionales, por encima de los límites máximos establecidos, podrá dar lugar a la modificación de la resolución de concesión (art. 12).

En cuanto al reintegro de las cantidades percibidas y del interés de demora correspondiente desde el momento del pago hasta la fecha en que se acuerde su procedencia, el art. 13.1 menciona los casos de nulidad y anulabilidad previstos en la Ley 38/2003, de 17 de noviembre, General de Subvenciones ${ }^{14}$ y los que a continuación se expresan, de conformidad con el art. 37 de esta Ley y el art. 112 de la Ley General de Hacienda: a) Obtención de la subvención falseando las condiciones requeridas para ello u ocultando aquéllas que lo hubieran impedido. b) Incumplimiento total o parcial del objetivo, de la actividad, del proyecto o la no adopción del comportamiento que fundamentan la concesión de la subvención. c) Incumplimiento de la obligación de justificación o la justificación insuficiente. d) Incumplimiento de la obligación de adoptar las medidas de difusión. e) Resistencia, excusa, obstrucción o negativa a las actuaciones de comprobación y control financiero, así como el incumplimiento de las obligaciones contables, registrales o de conservación de documentos cuando de ello se derive la imposibilidad de verificar el empleo dado a los fondos percibidos, el cumplimiento del objetivo, la realidad y regularidad de las actividades subvencionadas, o la concurrencia de subvenciones, ayudas, ingresos o recursos para la misma finalidad, procedentes de cualesquiera Administraciones o entes públicos o privados, nacionales, de la Unión Europea o de organismos internacionales. f) Incumplimiento de las obligaciones impuestas por la Administración a las entidades colaboradoras y beneficiarios, así como de los compromisos por éstos asumidos, con motivo de la concesión de la subvención, siempre que afecten o se refieran al modo en que se han de conseguir los objetivos, realizar la actividad, ejecutar el proyecto o adoptar el comportamiento que fundamenta la concesión de la subvención. g) Incumplimiento de las obligaciones impuestas por la Administración a las entidades colaboradoras y beneficiarios,

\footnotetext{
${ }^{13}$ Que el Decreto cita como "LGHPA".

${ }^{14}$ Que también cita el Decreto como "LGS".
} 
así como de los compromisos por éstos asumidos, con motivo de la concesión de la subvención, distintos de los anteriores, cuando de ello se derive la imposibilidad de verificar el empleo dado a los fondos percibidos, el cumplimiento del objetivo, la realidad y regularidad de las actividades subvencionadas, o la concurrencia de subvenciones, ayudas, ingresos o recursos para la misma finalidad, procedentes de cualesquiera Administraciones o entes públicos o privados, nacionales, de la Unión Europea o de organismos internacionales. h) La adopción, en virtud de lo establecido en los artículos 87 a 89 del Tratado de la Unión Europea, de una decisión de la cual se derive una necesidad de reintegro. i) En los demás supuestos previstos en la normativa reguladora de la subvención. El art. 13.2 añade también el reintegro del exceso obtenido sobre el límite máximo de ayuda permitido en el Anexo del Decreto, en el supuesto de obtención concurrente de ayudas.

VI.- A la publicidad y al control de las ayudas se dedican también varios preceptos. Así, las ayudas concedidas se han de hacer constar en la Base de Datos de Subvenciones y Ayudas Públicas de la Comunidad Autónoma ${ }^{15}$, recogiéndose los datos que se determinen en su norma reguladora (art. 9). En los supuestos de ayudas cofinanciadas por los Fondos estructurales, las normas de desarrollo del Decreto deberán cumplir las disposiciones sobre información y publicidad que se dicten por la Unión Europea (Disposición Final Segunda 3).

La vigilancia y control corresponde a la Administración de la Junta de Andalucía, que puede realizar las inspecciones y comprobaciones que resulten oportunas, así como recabar las informaciones pertinentes (art. 11). Por otra parte, los órganos de la Administración de la Junta de Andalucía, sus organismos autónomos y empresas responsables de la gestión de las líneas de ayudas que sean aprobadas han de trasladar a la Consejería de Presidencia para su presentación a la Comisión Europea un informe anual de ejecución de cada línea de ayudas (art. 14.1) ${ }^{16}$. Finalmente, las instancias anteriormente

15 Vid. la Orden de 25 de febrero de 2002, por la que se crea y regula la base de datos de subvenciones y ayudas públicas de la Comunidad Autónoma de Andalucía (BOJA de 7 de marzo de 2002).

${ }^{16}$ En el informe no se recogen las ayudas de minimis. No obstante, previa solicitud por escrito, el Centro Gestor debe facilitar a la Consejería de Presidencia para que lo traslade a la Comisión Europea toda la información que ésta considere necesaria para determinar si se han cumplido las condiciones establecidas en el Reglamento 1998/2006 y, especialmente, el importe total de la ayuda de minimis recibida por cualquier empresa (art. 14.2). 
citadas deben también trasladar a la Consejería de Presidencia, para su notificación a la Comisión Europea, cualquier proyecto individual de ayuda destinada a la inversión en el caso de que los costes subvencionables superen los veinticinco millones de euros y la ayuda exceda del equivalente bruto de subvención de cinco millones de euros (art. 15).

VII.- Por lo que respecta a la eficacia de lo dispuesto en el Decreto, hay que distinguir diferentes supuestos.

En primer término, y con carácter general, las ayudas previstas en el Decreto no se podrán llevar a efecto hasta que exista una Decisión de la Comisión Europea por la que se autorice el régimen y en los términos que se establezca conforme al art. $88 \mathrm{TCE}^{17}$. Sin perjuicio de ello, pueden llevarse a efecto las medidas que tengan carácter de ayudas de minimis y respeten las cuantías y condiciones establecidas en el Reglamento 1998/2006, de la Comisión (Disposición Transitoria Primera 1).

En cambio, pueden llevarse a efecto desde la entrada en vigor del Decreto las medidas que de forma clara no sean constitutivas de ayuda de estado por no cumplir con los requisitos señalados en el art. 87.1 TCE, entendiéndose por tales las que tengan por beneficiarios a personas físicas que no ejerzan actividad de contenido económico, por intervenir en el mercado sólo como consumidores individuales, y siempre y cuando que la ayuda se conceda sin discriminación por el origen de los productos (Disposición Transitoria Primera 2).

Finalmente, el Decreto entró en vigor el 8 de febrero del presente año, aunque es aplicable a las ayudas que se concedan a partir del 1 de enero.

${ }^{17}$ Informa la Exposición de Motivos del Decreto que el proyecto del mismo se remitió a la Comisión Europea y se registró como ayuda N 599/2006. El anterior Decreto 23/2001 fue autorizado para el periodo 2000-2006 mediante Decisión de 28 de noviembre de 2000. 